\title{
Prevalence of Stillbirth and Associated Factors among Immediate Postpartum Mothers at Bahir Dar, Felegehiwot Hospital, Northwest Ethiopia: Cross-sectional Study
}

\author{
Agerie Mengistie Zeleke ${ }^{1, *}$, Mulusew Andualem Asemahagn ${ }^{2}$ \\ ${ }^{1}$ Department of Midwifery, Teda Health Science College, Gondar, Ethiopia \\ ${ }^{2}$ Department of Epidemiology and Biostatistics, School of Public Health, College of Medicine and Health Science, Bahir Dar University, \\ Bahir Dar, Ethiopia
}

Email address:

ageriemengistie@gmail.com (A. M. Zeleke), muler.hi@gmail.com (M. A. Asemahagn)

${ }^{*}$ Corresponding author

\section{To cite this article:}

Agerie Mengistie Zeleke, Mulusew Andualem Asemahagn. Prevalence of Stillbirth and Associated Factors among Immediate Postpartum Mothers at Bahir Dar, Felegehiwot Hospital, Northwest Ethiopia: Cross-sectional Study. International Journal of Biomedical Engineering and Clinical Science. Vol. 7, No. 2, 2021, pp. 22-29. doi: 10.11648/j.ijbecs.20210702.12

Received: November 12, 2020; Accepted: December 2, 2020; Published: April 20, 2021

\begin{abstract}
Background: Stillbirth rates are among the indicators of maternal and child health care quality in the countries. However, the majority of neonatal deaths and almost all stillbirths were underreported in Ethiopia. Therefore, this study aimed to determine the prevalence of stillbirth and associated factors among immediate postpartum mothers in the study area. Methods: An institution-based cross-sectional study was employed at Felegehiwot comprehensive specialized hospital from March to May $2016(n=310)$. A pretested structured interviewer- administered questionnaire and medical chart reviews were used to collect data from immediate postpartum mothers. Data were entered into Epi Info version 7 and analyzed using SPSS version 20. The binary logistic regression model fitted to identify stillbirth; adjusted odds ratio (AOR) with a $95 \%$ confidence interval $(\mathrm{CI})$ was computed to assess the strength of association. Variables having less than 0.05 p-value of in the multivariable considered as factors associated with stillbirths. Result: The prevalence of stillbirth was 8.7\% (95\%CI: 5.8, 12.4). Rural dwelling ( $\mathrm{AOR}=2.86,95 \% \mathrm{CI}$ : 1.107 .47$)$, twin pregnancy $(\mathrm{AOR}=6.69,95 \% \mathrm{CI}: 1.77,25.2)$, medical or obstetrical illnesses during pregnancy $(\mathrm{AOR}=5.54,95 \% \mathrm{CI}$ : 1.71 17.94) and complications during labor and delivery $(\mathrm{AOR}=4.96,95 \% \mathrm{CI}: 1.48$ 16.58) were factors associated with stillbirths. Conclusion: This study revealed that the magnitude of stillbirth was high in rural dwelling, twin pregnancy, medical or obstetrical illnesses during pregnancy, and complicated labor were associated with increased occurrences of stillbirth. This finding suggests that particular emphasis on rural dwellers and strengthening quality of Maternal in pregnancy and labor delivery service and proper following during labor attending process are mandatory to reduce stillbirth.
\end{abstract}

Keywords: Stillbirth, Immediate Postpartum Mothers, Felegehiwot Referral Hospital

\section{Introduction}

The World Health Organization (WHO) defines stillbirth as a baby born with no signs of life at or after the age of viability (after 28 weeks of gestation) [1]. Stillbirths are the highest rates of all the adverse pregnancy outcomes and most common in developing countries [2]. Globally, about 4million stillbirths occurred every year, of which half-million stillbirths happened at the time of labor, of which also most cases occurred in developing countries [3]. In Sub-Saharan
Africa, stillbirths accounted for more than 3\% of all deliveries annually [4]. According to recent shreds of evidence, the average stillbirth rate in developing countries was found to be 26 per 1000 births, which is five times higher than in developed countries $[5,6]$. Stillbirths occurred during the period of perinatal, of which half of all stillbirths occurred in the intrapartum period of labor and delivery [7]. Stillbirth rates are among the indicators of antenatal and obstetric care quality at health facilities' [8].

Reports of stillbirths and mortality usually came from 
officially registered deaths; however, most of the cases remained under reported and majority of stillbirths registered as intrauterine deaths. Low institutional delivery and poor health information system are the main reasons for the underreporting of still birth and neonatal mortality in developing countries [9-11]. Moreover, isolation of mother and newborn during the postpartum period, the norm of acceptance to newborn death as normal in the community, and low utilization of vital registration in the country were also factors responsible for low reporting of stillbirth [12].

Characteristics like age, marital status, occupation and residence, parity, previous stillbirths, obstetrical infections, fetal presentations, and obstetric complications were determinants [13-15]. Health service factors (prenatal and intrapartum labor management) are also associated with stillbirth and other adverse birth outcomes [10]. Interventions like focused antenatal care (ANC), enhancing institutional delivery, and providing $\mathrm{MCH}$ services free of charge were designed to reduce stillbirth incidence and occurrence. Thought different studies conducted to assess the rate of stillbirth [17], there is scarcity of information in the study area regarding the magnitude and determinants of stillbirths.

Hence, this studies important to reduce adverse birth outcomes through evidence-based interventions in the health care system.

\section{Methods}

\subsection{Study Setting}

The study was conducted in Bahir Dar town Amhara regional state, northwest Ethiopia. It is the capital city of Amhara regional state and is located $565 \mathrm{Km}$ from Addis Ababa in northwest Ethiopia. According to the Amhara Bureau of finance and economic development 2016 projection, the town hosts 277,566. There are six governmental health centers, one comprehensive specialized hospital, and five higher private clinics in this town. Regarding maternal health services of Felegehiwot comprehensive specialized hospital has obstetrics, high risk, and gynecology wards used for pregnant women admission and treatment. It serves more than five million people in the catchment area and neighboring regions, and according to the hospital statistics office report, on average, about 6000 deliveries reported annually.

\subsection{Study Design and Period}

A facility-based cross-sectional study design was employed at Bahir Dar Felegehiwot comprehensive specialized hospital from March 10 to May 10, 2016.

\subsection{Population and Sample}

All participants who gave birth after 28 weeks of age completed at Felegehiwot comprehensive specialized hospital were the source the population for this study.

The participants were determined using a single population proportion formula. The following assumptions of the percentage of stillbirth from previous studies were $7.1 \%$ $(P=0.071)[16], 3 \%=0.03)$ margin of error, $95 \%$ confidence interval $(\mathrm{CI})$ and took a $10 \%$ no-response rate. The final 310 study participants selected by systematic random sampling technique.

\subsection{Data Collection Procedure}

Initially, the survey was prepared in English, and then translated to the local language (Amharic) and back to English by a language expert to check its consistency. Data about maternal socio- demographic, clinical, and obstetrical attributes collected through interviewer-administered structured questionnaire and chart reviews. Three midwives for data collection and supervision were recruited, and oneday training was given on how the study's objective and how to collect it from immediate postpartum mothers in stable conditions after delivery and before leaving the maternity room. Daily, the principal investigator and supervisor checked completeness and consistency of collected questionnaires.

\subsection{Operational Definitions}

Obstetric labor complications are difficulty or abnormality that arises during labor and delivery [17] Medical or obstetrical illnesses: when medical diseases or obstetrical illnesses occurred during pregnancy time.

Low birth weight: when a newborn weight is below 2500 grams.

Preterm: when a birth takes place before the expected due date that 36 weeks of gestational age.

\subsection{Data Processing and Analysis}

Data were coded and entered into Epi Info version 7 and exported to SPSS version 20 for further processing and analysis. Descriptive statistics such as mean, median, proportions were carried out, and text, graph, and tables were used to present the data. The binary logistic regression model was fitted to identify factors associated with stillbirth. Crude and Adjusted Odds ratio with95\% CI were used to describe the strength of association between outcome and predictor variables.

Variables with a p-value of less than 0.05 in the multivariable model considered as statistically significantly associated with stillbirth.

\subsection{Ethical Considerations}

Ethical approval was obtained from the ethical committee of the College of Medicine and Health Sciences, Bahir Dar University. A letter of permission was also obtained from Amhara Regional State Health Bureau and Felegehiwot Comprehensive Referral Hospital. Verbal consent was used because of the low literacy level in the community as well as some participants are uncomfortable to sign form. Similarly; the participants were informed about the purpose of the study. Participants were also informed on data confidentiality issues, on a voluntary basis and they can refuse from the study at 
any time if they are not comfortable on the questionnaire at any steps.

\section{Results}

\subsection{Socio-demographic Characteristics}

A total of 310 immediate postpartum mothers were

Table 1. Socio-demographic characteristic of mothers who gave birth at Bahir DarFelegehiwot Referral Hospital, 2016 (n=310).

\begin{tabular}{|c|c|c|}
\hline Characteristics & Frequency & Percentages \\
\hline \multicolumn{3}{|l|}{ Age } \\
\hline$<20$ & 15 & 4.8 \\
\hline $20-34$ & 261 & 84.8 \\
\hline$>35+$ & 34 & 11 \\
\hline \multicolumn{3}{|l|}{ Marital status } \\
\hline Married & 274 & 88.4 \\
\hline Single & 36 & 11.6 \\
\hline \multicolumn{3}{|l|}{ Residence } \\
\hline Rural & 122 & 39.4 \\
\hline Urban & 188 & 60.6 \\
\hline \multicolumn{3}{|l|}{ Educational status } \\
\hline No formal education & 119 & 38.4 \\
\hline Primary and secondary school & 105 & 33.9 \\
\hline College and above & 86 & 27.7 \\
\hline \multicolumn{3}{|l|}{ Income level in ETB } \\
\hline$<1500$ & 167 & 53.9 \\
\hline $1500-2500$ & 80 & 25.8 \\
\hline$>2500$ & 63 & 20.3 \\
\hline \multicolumn{3}{|l|}{ Religion } \\
\hline Orthodox & 270 & 87.1 \\
\hline Muslim & 37 & 11.9 \\
\hline Others & 3 & 0.9 \\
\hline \multicolumn{3}{|l|}{ Occupation } \\
\hline Housewife & 214 & 68.9 \\
\hline Employed & 90 & 29.1 \\
\hline Other & 6 & 2.2 \\
\hline
\end{tabular}

\subsection{Obstetrics Related Characteristics}

More than half (56.5\%) of mothers were multiparous, most (84.6\%) had inter parity interval of $\geq 2$ years, $91 \%$ had at least one ANC follow-up, and $89 \%$ of pregnancies were also wanted, planned and supported. Nearly half (47.4\%) of mothers had at least one confirmed medical or obstetrical illnesses of which, $26.5 \%, 15.4 \%, 12.8 \%$, and $12.1 \%$ were pregnancy-induced hypertension, ante partum hemorrhage, Rh-immunization, and premature rupture of membrane (PROM), respectively. In addition, nearly three-fourth $(72.5 \%)$ of the mothers were delivered at term (after completed 37 weeks), 16.2\%were preterm, and the remaining were post-term gestational age at delivery. Historically, 11\% of women experienced a stillbirth in the past births (Table 2).

Status o labor, delivery and birth outcomes

The majority $(89.0 \%)$ of labor initiated spontaneously, of which 53.5\% mothers gave birth through spontaneous vaginal delivery, $38.4 \%$ were cesarean section mode, $93.9 \%$ included with the response rate of $100 \%$. The mean (SD) age of mothers was $26.6( \pm 5)$ years, most $(94.5 \%)$ of them were married, $60.6 \%$ were urban dwellers, and $68.1 \%$ of mothers were housewives. More than one-third (38.4\%) of respondents had no formal educational status, and the majority (87.1\%) were Christians (Table 1). 
Table 2. Obstetrics and related characteristics of mothers who gave birth at Bahir Dar Felegehiwot comprehensive specialized hospital, 2016 ( $n=310)$.

\begin{tabular}{|c|c|c|}
\hline Characteristics & Frequency & Percentages \\
\hline \multicolumn{3}{|l|}{ Condition of current Pregnancy } \\
\hline Planned, wanted and supported & 264 & 85.2 \\
\hline Unplanned & 46 & 14.8 \\
\hline \multicolumn{3}{|c|}{ Current pregnancy birth interval $(\mathrm{n}=175)$} \\
\hline$<2$ years & 27 & 15.4 \\
\hline$\geq 2$ years & 148 & 84.6 \\
\hline \multicolumn{3}{|l|}{ Parity } \\
\hline 1 & 140 & 45.2 \\
\hline $2-4$ & 138 & 44.5 \\
\hline$>5+$ & 32 & 10.3 \\
\hline \multicolumn{3}{|l|}{ Previous history of stillbirth } \\
\hline Yes & 34 & 11.0 \\
\hline No & 276 & 89.0 \\
\hline \multicolumn{3}{|l|}{ Gestational age in weeks } \\
\hline Term & 225 & 72.5 \\
\hline Preterm term & 49 & 16.2 \\
\hline Post-term & 36 & 11.6 \\
\hline \multicolumn{3}{|l|}{ ANC follow up } \\
\hline Yes & 282 & 91.0 \\
\hline No & 28 & 9.0 \\
\hline \multicolumn{3}{|l|}{ Obstetric complications } \\
\hline No & 163 & 52.6 \\
\hline Yes & 147 & 47.4 \\
\hline \multicolumn{3}{|c|}{ Obstetric and medical illness ( $\mathrm{n}=147$ ) } \\
\hline Pregnant induced Hypertension & 38 & 26.2 \\
\hline Hydrations & 13 & 7.4 \\
\hline Gestational diabetes mellitus & 5 & 3.4 \\
\hline Ante partum haemorrhage & 20 & 15.4 \\
\hline RH-factor & 19 & 12.8 \\
\hline HIV & 13 & 8.8 \\
\hline A nemia & 9 & 5.4 \\
\hline PROM & 18 & 12.1 \\
\hline Previous caesarean section & 9 & 6.0 \\
\hline Others* & 11 & 4.0 \\
\hline
\end{tabular}

Note: Others* chorioaminitis, IUGR (intrauterine growth restriction)

Table 3. Labor and delivery related characteristics of mothers and birth outcome at Bahir Dar Felegehiwot Comprehensive Specialized Hospital, 2016.

\begin{tabular}{lll}
\hline Characteristics & Frequency & Percentage \\
\hline Labour complications & 163 & 52.6 \\
Yes & 147 & 47.4 \\
No & & 15.5 \\
Labor complications (n=163) & 50 & 16.1 \\
Non reassuring fetal heart rate pattern & 48 & 8.1 \\
Prolonged labour & 25 & 3.5 \\
Mal presentation & 11 & 2.6 \\
Obstructed labour & 8 & 3.2 \\
Malposition & 10 & 3.6 \\
Uterus rupture & 11 & 89.0 \\
Others* & & 11.0 \\
Status of labour & 276 & 63.9 \\
Spontaneous & 34 & 42.1 \\
Induced /augmented & & \\
Sex of newborn & 196 & 38.4 \\
Male & 114 & 8.1 \\
Female & & 53.5 \\
Mode of delivery & 119 & 23.5 \\
Caesarean section & 25 & 72.3 \\
Instrumental & 166 & 4.2 \\
Spontaneous vaginal delivery & & \\
$<2500$ gram & 73 & 224 \\
$25000-4000$ gram & 13 & \\
$\geq 4000$ gram & & \\
\hline
\end{tabular}

Note: others*. Cord prolapsed, chorioaminitis. 


\subsection{Factors Associated with Stillbirth}

From the binary logistic regression analysis factors like rural dwelling, multiple pregnancies, illness during pregnancy, labor complications were significantly associated with a stillbirth at $0.05 \mathrm{p}$-value in the multivariable analysis. Thus, women who were rural dwelling, the odds of stillbirth were 2.86 times higher than those in urban dwellers $(\mathrm{AOR}=2.86,95 \% \mathrm{CI}: 1.107 .47)$.

Those mothers who had confirmed medical or obstetrical illnesses during pregnancies found the odds of stillbirth were 5.54timeshigherthanthosewhohadnosuchhistory ( $\mathrm{AOR}=5.54$, 95\% CI: 1.71-17.94). Similarly, women who had twin pregnancies, the odds of Stillbirths were 6.69 times higher than those who had singleton $(\mathrm{AOR}=6.69,95 \% \mathrm{CI}$ : 1.7725 .2$)$. Moreover, those who had complications during labor and delivery, the odds of stillbirth were 4.96 times higher than those who had no such events $(\mathrm{AOR}=4.96,95 \% \mathrm{CI}$ : 1.48 16.58) (Table 4).

Table 4. Bi-variable and multivariable logistic regressions on different variables towards stillbirth in postpartum mother who gave birth at Felegehiwot Referral Hospital, $2016(n=310)$.

\begin{tabular}{|c|c|c|c|c|}
\hline \multirow{2}{*}{ Variables } & \multicolumn{2}{|c|}{ Stillbirth } & \multirow{2}{*}{ Crud OR (95\% CI) } & \multirow{2}{*}{ Adjusted OR (95\%CI) } \\
\hline & Yes & No & & \\
\hline \multicolumn{5}{|l|}{ Age of mother } \\
\hline $18-34$ & 18 & 255 & 1 & 1 \\
\hline $35+$ & 9 & 28 & $4.55(1.8611 .05)$ & $3.27(0.9211 .61)$ \\
\hline \multicolumn{5}{|l|}{ Sex of newborn } \\
\hline Male & 21 & 175 & 1 & 1 \\
\hline Female & 6 & 108 & $0.46(0.181 .18)$ & $0.44(0.151 .29)$ \\
\hline urban & 9 & 179 & 1 & 1 \\
\hline Rural & 18 & 104 & $3.44(1.497 .94)$ & $2.86(1.107 .47)^{*}$ \\
\hline \multicolumn{5}{|l|}{ Mode of delivery } \\
\hline Vaginal & 15 & 176 & 1 & 1 \\
\hline Cesarean section & 12 & 102 & $1.34(0.592 .91)$ & $0.41(0.131 .27)$ \\
\hline \multicolumn{5}{|c|}{ Illness during pregnancy } \\
\hline No & 5 & 158 & 1 & 1 \\
\hline Single & 21 & 270 & 1 & 1 \\
\hline Twin & 6 & 13 & $5.93(2.04$ 17.20) & $6.69(1.7725 .2)^{*}$ \\
\hline \multicolumn{5}{|l|}{ Parity } \\
\hline Primiparous & 9 & 131 & 1 & 1 \\
\hline Multiparous & 13 & 125 & $1.51(0.623 .66)$ & $1.24(0.433 .51)$ \\
\hline Grand multiparous & 5 & 27 & $2.69(0.838 .67)$ & $0.51(0.092 .85)$ \\
\hline \multicolumn{5}{|l|}{ Labor complications } \\
\hline No & 6 & 138 & 1 & 1 \\
\hline Yes & 21 & 145 & 3.33 (1.30 8.49) & $4.96(1.4816 .58)^{*}$ \\
\hline \multicolumn{5}{|l|}{ History of stillbirth } \\
\hline No & 20 & 255 & 1 & 1 \\
\hline Yes & 7 & 28 & $3.18(1.238 .20)$ & $1.98(0.547 .23)$ \\
\hline
\end{tabular}

* Showed statistical significance at 0.05 p-value

\section{Discussion}

The prevalence of stillbirth in this study was $8.7 \%$, with (95\% CI: $5.8 \% 12.4 \%$ ). This finding was consistent with the results from hospitals in Ethiopia ranged from $6 \%$ to $7.72 \%$ $[8,9,12,18]$. However, this finding was higher than results from Tanzania (3.6\%) [19], Zambia (3.4\%) [14], Ghana $(2.39 \%)$ [20], Malawi (3.6\%) [21], Nigeria (2.34\%) [22], and Gamo Gofa, Southern Ethiopia (1.9\%) [23]. Also, this finding was higher than the EDHS 2011 report of $1.8 \%, 11.7$ per 1000 births EDHS 2016 [24], and different systematic reviews from sub-Saharan A African countries of the prevalence of stillbirth ranged from 2.1 to $3.3 \%[3,25]$. The possible explanations for the observed variations might be the quality of health services, socio-cultural factors, and health-seeking behavior. Besides, there were methodological differences between studies where a community- based study underestimated the prevalence and was often characterized by under-reporting of stillbirth in the community. Furthermore, most of the referral and specialized hospitals' deliveries are complicated and transferred from other primary health facilities that might increase the probabilities of stillbirths. Despite, better health services and highly skilled professionals in the hospitals, delays from mother's healthseeking behaviors and poor referral systems in the lower health facilities contributed to the increased rate of stillbirth in the health facilities.

This study also revealed that the magnitude of adverse birth outcomes was $30.6 \%$, and the rate of cesarean section mode of delivery was $38.4 \%$, which is higher than the WHO recommendation of $5-15 \%$. The magnitude of adverse birth outcomes was consistent with those findings in Wollo, 
Ethiopia (27.5\%) [12]. However, this study result was higher than previous studies of $24.5 \%$ Hosanna [22] and $23 \%$ in Gondar, Ethiopia [16]. These could be due to sociodemographic variations, health-seeking behaviors, and clinicians' professional expertise for managing labor and delivery at health facilities.

This study also revealed that rural dwelling, medical or obstetrical illnesses, multiple pregnancies, and obstetric complications during labor and delivery were predictors of stillbirth. Thus, those mothers who came from rural areas had a three times higher probability of stillbirth than urban residents. This finding was consistent with those studies from Zimbabwe [26] and Ghana [20]. Rural areas are often associated with low quality of health services, including and maternal health care. Besides, most mothers were referred from remote areas with inadequate monitoring of labor during ambulance travel.

Women who had confirmed medical or obstetrical illnesses during pregnancy were associated with stillbirth occurrence compared to those who had no such history. This finding was in line with previous studies in Nigeria and Ethiopia [16, 22, 27]. These could be because of diseases such as infections of HIV, anemia, and APH are often associated with increased stillbirth.

On top of that, complications during labor and delivery associated with increased stillbirth occurrence compared to those women who had normal labor and delivery. This finding was also comparable with the study done in Mekelle, Ethiopia [9].

Complications during labor and delivery might induce intra-uterine oxygen inadequacy, which leads to fetal distress, which leads to stillbirth. This finding was in line with another result in India, and Gambia [28, 29].

About $59.7 \%$ stillbirths occurred during the intrapartum period, which can be prevented through appropriate intrapartum care during labor and prompt treatment of complications like preterm labor, the prolonged second stage of labor, and mechanical factors. In addition, non reassuring fetal heart rate had a significant association with stillbirth outcomes. These results are supported by a systematic review done from sixteen hospitals and community based prenatal mortality studied in Ethiopia [3, 6] and agrees with other reports $[11,30,31]$. Therefore, identification and management of the complications and provision of care by skilled healthcare providers avail ability and utilization of comprehensive emergency obstetric care may improve and keys to reducing stillbirths.

Consequently, there is a need to reinforce and strengthen health workers' abilities to accurately monitor women in labor at all levels of care for early identification and management of complications. This includes early referral if a woman is at a health center has complications during antenatal follow up and labor delivery process.

Likewise, this study also revealed that multiple pregnancies were associated with an increased stillbirth occurrence compared to singleton birth. The reason could be intrauterine growth restrictions and congenital disabilities and mechanical factors that are common in multiple pregnancies, which increases the vulnerability of stillbirth. In addition, multiple pregnancies require particular medical interventions, and providing quality of health service that are more accessible and available in our study area. This result is in agreement with findings in northwest Ethiopia [13] and other studies $[8,9,18]$.

\section{Limitations}

Firstly, since it was based on an institutional study, it is difficult to generalize the community as the study was in a referral hospital. It may overestimate the stillbirth rate and not show the real picture of these stillbirth outcomes areas. Secondly, this study also shared the limitations of crosssectional studies and hence may not be possible to establish a cause-effect relationship between stillbirth and explanatory variables.

\section{Conclusions}

The magnitude of stillbirth in this study was high. Rural dwelling, twin pregnancy, medical or obstetrical illnesses during pregnancy, and complicated labor associated with increased stillbirth. This finding suggests that particular emphasis on rural dwellers and strengthening quality of $\mathrm{MCH}$ service and proper management of labor is mandatory to reduce stillbirth.

\section{Declarations}

\section{Abbreviations}

ANC: Antenatal Care, AOR: Adjusted Odds Ratio, CI: Confidence Interval, EDHS, Ethiopia Demography and Health Survey, IUFD: Intra-Uterine Fetal Death, PROM: Premature Rupture of Membrane, SD Standard Deviation, and SPSS: Statistical Package for Social Science, WHO: World Health Organization

\section{Consent for Publication}

Not applicable.

\section{Availability of Data and Material}

Data will be shared upon reasonable request from the corresponding author.

\section{Authors' Contributions}

AM conceived the study. AM, and MA, equally participated in the design, data collection, statistical analysis and writing-up of the manuscript. All authors read and approved the final manuscript

\section{Opposed Reviewers}

There is no opposed reviewer for this manuscript except the authors of it: Agerie Mengistie (MSc), Lecturer, Teda 
Health Science College, Gondar, Ethiopia; and Mulusew Andualem Asemahagn (PHD), Lecturer, School of Public Health, College of Medicine and Health Science, Bahir Dar University, Bahir Dar, Ethiopia.

\section{Study's Contributions}

Prevalence of stillbirth and determinates factors is still challenge and has not reduced, in Ethiopia. As result it needs, credible evidence on the status of Prevalence of stillbirth and its associated factors. Hence, institutional based researches on immediate postpartum mothers are very important to generate baseline information for policies makers in the health systems and inform researchers for further study. Moreover, findings of cross-sectional studies are important for new design approach for providing maternal and child health services. Therefore, this study was aimed to assess the prevalence of stillbirth and associated factors among immediate postpartum mothers at Felegehiwot comprehensive specialized hospital, northwest, Ethiopia.

\section{Funding}

No funding was obtained.

\section{Competing Interest}

The authors declared that they have no competing interests.

\section{Acknowledgements}

We would like to thank Bahir Dar University, College of Medicine and Health Science School of nursing for the opportunity given to us to conduct this title. We would like to thank the school of nursing for their support by writing a letter of cooperation for Feleg Hiwot Referral Hospital to get the data. We would like to thank study participants, data collectors, and supervisors for their collaboration. Finally, we would like also to acknowledge gynecologists and obstetricians who are working in Felege Hiwot Referral Hospital for their unreserved support.

\section{References}

[1] Ntuli T., HN. M. An Investigation of the Stillbirths at a tertiary Hospital in Limpopo Province of South Africa. Canadian Center of Science and Education. 2012; 4.

[2] Robert M., Silver MD., Michael W., Varner MD., etail. WorkUp of Stillbirth: A Review of the Evidence. NIH Public Access. 2009.

[3] Say L., Donner A., Gülmezog M.,. e. The prevalence of stillbirths: a systematic review. Open Access. 2006; 3: 1-11.

[4] Manandhar SR., Ojha A., Manandhar DS., etal. Causes of Stillbirths and Neonatal deaths in Dhanusha district, Nepal: A verbal autopsy study. Kathmandu University Medical. 2010; 1 $(29,62-72)$
[5] Mcclure M., Pasha O., Shivaprasad S., etal. Epidemiology of stillbirth in low-middle income countries. NIH Public Access. 201112 1379-85.

[6] Alison G ÖT, Ann-B, etail Global causes of maternal death: a WHO systematic analysis

[7] WHO, UNICEF, MOH, UNFPA, The World Bank and the United Nations Population Division. Trends in maternal mortality: 1990 to 2013. 2014.

[8] Berhan Y. BA. Perinatal Mortality Trends in Ethiopia. Ethiop J Health Sciences. 2014; http://dx.doi.org/10.4314/ejhs.v24i1.4S.

[9] Berhe H. BH, Zeleke D. Factors Associated with Patterns of Birth outcome at Public Hospitals In Mekelle Town, Tigray Region, Ethiopia: A Case-Control Study. Innovative Association. 2013; 2: 67-81.

[10] Feyissa R. GA. Determinants of institutional delivery among childbearing age women in western ethiopia unmatched case control study. Plos one | wwwPlosoneOrg. 2014; 9 (597194).

[11] Frøen J., Sanne S., Aleem A., etal. Making stillbirths count, making numbers talk - Issues in data collection for stillbirths. BMC Pregnancy and Childbirth. 2009; 9: 58: 2-6.

[12] Eshete AS., Birhanu D., B. W. Birth outcomes among Laboring Mothers in Selected Health Facilities of North Wollo Zone, Northeast Ethiopia.

[13] Bahir Dar, Ethiopia. 2013; 5: 7 , (http://dx.doi.org/10.4236/health.2013.57154).

[14] Bayou G., Y. B. Perinatal Mortality and Associated Risk Factors. Ethiop J Health Sci. 2012; 22: 3: 153-4.

[15] Turnbull E., Mwila K., Lembalemba M., etal. Causes o f Stillbirth, Neonatal Death And Early Childhood Death In Rural Zambia by Verbal Autopsy Assessments. Tropical Medicine and International Health. 2011; 16 (894-901).

[16] Yaya Y., Eide T., Norheim F., etal. Maternal and Neonatal Mortality in Bonke woreda, South-West Ethiopia: Estimates and Socio-Economic Inequality.. PLoS ONE 9. 2013; 4: 96294 (et001-007): doi: 10.1371.

[17] Adane A., Ayele A., Ararsa GE., etail. Adverse birth outcomes among deliveries at Gondar University Hospital, Northwest Ethiopia. Open Access. 2014; 14: 2-8.

[18] DeFranco EA. SD, Boslaugh SE, et al. obstetrics and gynecology. World Health Organazition (WHO). 2012; 3: 264

[19] y. G. Assessment of Factors Associated with Perinatal Mortality among Public Hospital Deliveries in Addis Ababa, Ethiopia. Addis Ababa University. 2015: 1-51.

[20] LöfwanderM. Stillbirths and Associations with maternal education from a regional hospital in north Eastern Tanzania. University of Tromsø, Norway. 2012.

[21] BG K. Risk factors associated with stillbirth. a case study of the hohoe municipality, ghana. Kwame Nkrumah University of Science and Technology. 2011: 1-102.

[22] Lily C, JohanneS., J. O. Factors Associated with Perinatal Deaths in Women Delivering in a Health Facility in Malawi. Journal of Research in Nursing and Midwifery (JRNM). 2012; 1: 5: 69-79. 
[23] Audu BM., Alhaji M., Takai U., etail. Risk Factors for Stillbirths at Universty of Maiduguri Teaching Hospital, Maiduguri, Nigeria: A Cross-Sectional Retrospective Analysis. Niger Med J2009; 50: 2.

[24] Gebremedhin G., AbrahaT., Wassie B., etal. Incidence of Low Birth Weights Babies and Associated Factors among Post Partum Mothers Who Gave Birth at Felegehiowt Referral Hospital, Bahridar, Ethiopia. World Journal of Pharmacy and Pharmaceutical Sciences. 2015; 4 (2278-4357).

[25] World Health Organazition. Addis Ababa EIIC, Maryland, USA. Ethiopia Mini Demographic and Health Survey. WHO Addis Ababa, Ethiopia 2014.

[26] Serie. L. Stillbirths an Executive Summary for the Lancet Series. Health Systems Research Unit 2011; 10.

[27] Tachiweyika E., Gombe N., Shambira G, etail. Determinants of perinatal mortality in Marondera district, Mashonaland East Province of Zimbabwe. A case control study. Pan African Medical. 2011; 8: 7.

[28] Andargie G., Berhane Y., Worku AA., Y. K. Predictors of perinatal mortality in rural population of Northwest Ethiopia: a prospective longitudinal study. BMC Public 2013; 13: 14712458.

[29] Behal M., R. V. Maternal Risk Factors For Perinatal Mortality in india. Indian Journal of Obstetrics and Gynecology Research. 2015; 2 (6): 92-9.

[30] Jammeh AB. VS, Sundby J. Stillbirths in Rural Hospitals In the Gambia. Bmc Pregnancy and Childbirth2014; 12: 109.

[31] Gizaw M. MM, Mekonnen W. Trends and risk factors for neonatal mortality in Butajira District, South Central Ethiopia. BMC Pregnancy and Childbirth2014; 14: 64. 